\title{
EXÁMENES PREOPERATORIOS DE RUTINA EN CIRUGÍA ELECTIVA: ¿CUÁL ES LA EVIDENCIA?*
}

\author{
Dr. Claudio Nazar J. ${ }^{1}$, Int. Javier Bastidas E. ${ }^{2}$, Dr. Guillermo Lema F. ${ }^{1}$
}

1 División de Anestesiología.

2 Interno de Medicina.

Escuela de Medicina, Facultad de Medicina, Pontificia Universidad Católica de Chile.

Santiago, Chile.

\begin{abstract}
\section{Routine preoperative testing in elective surgery: what is the evidence?}

Any patient, who is undergoing a surgical or invasive procedure, requiring anesthesia or sedation, needs a preoperative evaluation. This is essential in any type of surgical procedure. Most of the times, clinical evaluation is sufficient to establish perioperative as well as patient's risk factors. Some of them may be modified during the period close to the intervention, without supplementary testing or consultation to others specialists. Currently, the tendency of specialists is request "routine preoperative testing" sometimes without a previous analysis of the clinical history and physical examination. It has been shown that a healthy young patient, undergoing elective surgery, does not need any test before surgery. Preoperative tests increases health costs and don't significantly change perioperative patient's morbidity and mortality. Tests such as chest X-ray, electrocardiogram, hematocrit/hemoglobin, coagulation test, platelet count, blood glucose and pregnancy tests have precise indications and should not routinely be requested to all the surgical population.
\end{abstract}

Key words: Routine diagnostic tests, morbidity, mortality, surgical procedures, perioperative period.

\section{Resumen}

Todo paciente que se va a someter a un procedimiento quirúrgico, con anestesia, necesita una evaluación preoperatoria, siempre importante para el proceso quirúrgico. La mayoría de las veces, la evaluación clínica es suficiente para establecer el riesgo perioperatorio y determinar los factores de riesgo del paciente susceptibles de ser modificados durante el período cercano a la intervención, sin necesidad de recurrir a exámenes de apoyo complementarios ni interconsultas a especialistas. En la actualidad, la tendencia de los especialistas es solicitar los llamados exámenes preoperatorios "de rutina", muchas veces sin un análisis previo de la historia clínica y examen físico. Se ha demostrado que un paciente joven y sano, sometido a una cirugía de forma electiva, no necesita de exámenes previos a la intervención quirúrgica. Estos exámenes preoperatorios aumentan los

*Recibido el 13 de noviembre de 2013 y aceptado para publicación el 9 de diciembre de 2013.

Los autores no refieren conflictos de interés.

Correspondencia: Dr. Claudio Nazar J.

Marcoleta 367, Santiago, Chile. Fax: 26327620. CP: 6510260

cenazar@med.puc.cl 
costos en salud y casi nunca cambian significativamente la morbimortalidad perioperatoria. Exámenes como radiografía de tórax, electrocardiograma, hematocrito/hemoglobina, pruebas de coagulación, recuento de plaquetas, glicemia y test de embarazo tienen indicaciones precisas y no deben solicitarse rutinariamente a toda la población quirúrgica.

Palabras clave: Exámenes preoperatorios de rutina, morbilidad, mortalidad, intervención quirúrgica, período perioperatorio.

\section{Introducción}

La "American College of Physicians" recomienda que los exámenes de laboratorios preoperatorios se soliciten con criterio selectivo y justificación clínica. Sin embargo, muchas veces se solicitan exámenes de forma rutinaria, muchos de los cuales terminan siendo innecesarios. Generalmente, o en su gran mayoría, se obtienen resultados alterados que no modifican la conducta y generan un dilema de qué hacer frente a ellos. A veces, se solicitan como respaldo médico-legal, sin embargo, más grave sería omitir información que ya obtuvimos y que podría terminar siendo relevante en la práctica clínica. Entre 30 y $95 \%$ de los exámenes preoperatorios alterados no son anotados en la ficha médica del paciente, por lo que no influirán en la conducta preoperatoria tanto del cirujano como del anestesiólogo ${ }^{1}$.

"Si no lo va a usar, no lo pida". Por otro lado, se aumentan innecesariamente los costos en salud y, en ocasiones, se posterga un procedimiento quirúrgico innecesariamente ${ }^{2}$.

El objetivo principal de la evaluación preoperatoria es identificar los factores de riesgo modificables del paciente y optimizar los cuidados perioperatorios, para lograr una significativa disminución en la morbimortalidad perioperatoria.

En el año 1976, Olsen y cols, concluyeron en un estudio con más de 1.500 pacientes que fueron sometidos a exámenes preoperatorios de rutina, que no existen diferencias en la morbimortalidad perioperatoria si estos son solicitados o no ${ }^{3}$. En el mismo año, Durbridge y cols, demostraron, utilizando más de 8.000 exámenes preoperatorios realizados en la admisión del hospital, que no hay beneficios en la morbimortalidad del paciente ni en la estadía hospitalaria si estos son realizados rutinariamente ${ }^{4}$. En el año 1991, Narr y cols, demostraron que no hay cambios en la morbimortalidad perioperatoria en pacientes con clasificación American Society of Anesthesiologists (ASA) I al omitir los "exámenes de rutina”, por lo que desde ese año en la Clínica Mayo ya no se solicitan exámenes preoperatorios rutinarios en pacientes ASA I, disminuyendo así los costos y mejorando la eficiencia del sistema ${ }^{5}$. En el año 1997, Munro y cols, también señalaron que no existe evidencia que apoye el uso de exámenes preoperatorios llamados de rutina ${ }^{6}$. En el año 2012, el National Institute of Clinical Excellence ${ }^{7}$, realizó una larga recomendación sobre los exámenes preoperatorios necesarios para cirugías electivas, la cual es de tediosa lectura y difícil aplicación en la práctica quirúrgica.

El objetivo de esta revisión es buscar la evidencia existente en relación a los exámenes preoperatorios llamados "de rutina" y realizar una recomendación de fácil aplicación para el cirujano general y subespecialista.

\section{Radiografía de Tórax (RxTx)}

La evidencia que existe actualmente no es de buena calidad y se basa sólo en opiniones de expertos con bajo nivel de evidencia médica. Las complicaciones pulmonares en el período postoperatorio ocurren entre 2,7 y $58,3 \%$ de los pacientes sometidos a cirugía. Sin embargo, no hay estudios que demuestren una asociación significativa entre una RxTx alterada y las complicaciones postoperatorias clásicamente descritas ${ }^{8}$. La recomendación actual es realizar una RxTx sólo si hay síntomas o signos cardiopulmonares de reciente aparición o si existe alguna condición clínica de insuficiencia cardíaca y/o pulmonar significativa ${ }^{1,9}$. La RxTx podría estar alterada en pacientes de edad avanzada ( $>65$ años), tabáquicos crónicos, con infección del tracto respiratorio reciente, enfermedad pulmonar obstructiva crónica (EPOC) y enfermedades cardíacas crónicas con relativa estabilidad en los últimos años. Dado lo anterior, no es necesario solicitar una nueva imagen porque muy probablemente se encuentren los mismo hallazgos que en exámenes anteriores, no conduciendo a cambios importantes en la conducta perioperatoria, generando más riesgos secundarios a la irradiación que beneficios en el paciente ${ }^{1,10}$.

\section{Electrocardiograma (ECG)}

Hepner y cols $^{11}$, señalaron que era aconsejable realizar un ECG de rutina en pacientes de edad avanzada ( $>65$ años) ya que al menos un cuarto de los adultos mayores pudiesen tener infartos asintomáticos. Un ECG alterado podría modificar la clasificación de estado físico de la ASA $^{12}$ de un paciente 
quirúrgico $\mathrm{y}$, además, permitiría tener un examen basal en caso de complicaciones cardiovasculares en el período postoperatorio inmediato. Sin embargo, la $\mathrm{ASA}^{10}$ sugiere que no se puede utilizar sólo la edad como criterio para realizar un ECG preoperatorio, teniendo mayor evidencia para su solicitud la existencia de enfermedades cardiovasculares y pulmonares previas a la cirugía, los factores de riesgo cardiovasculares del paciente y el tipo de cirugía a la que este se someterá. Un estudio realizado por De Sousa y cols ${ }^{13}$, en el año 2013, concluyó que en los pacientes en los cuales se realizó un ECG de rutina, se cambió la conducta perioperatoria en menos del $1 \%$ de los pacientes.

Por lo tanto, nuestra recomendación es solicitar un ECG en los siguientes pacientes: aquellos con signos o síntomas significativos de enfermedad cardiovascular y/o pulmonar, sometidos a cirugía de alto riesgo quirúrgico, sometidos a cirugía de riesgo quirúrgico intermedio con al menos 1 punto en el Revised Cardiac Risk Index (RCRI) o pacientes con 2 puntos en el RCRI, independiente del riesgo de la cirugía (Tabla 1 y 2$)^{9,14,15}$.

\section{Hematocrito/Hemoglobina (Hcto/Hb)}

En la actualidad, no existe ninguna guía o recomendación que avale el uso rutinario de $\mathrm{Hcto} / \mathrm{Hb}$ como examen preoperatorio. En primer lugar, en pacientes sometidos a cirugía de bajo riesgo quirúrgico ${ }^{14}$ menos del $1,5 \%$ de los pacientes tenía alteraciones en el $\mathrm{Hcto} / \mathrm{Hb}$ y sólo un $0,1 \%$ tenía alguna alteración que podría haber cambiado la conducta pre o intraoperatoria ${ }^{13}$. Por otro lado, en pacientes de bajo riesgo quirúrgico el uso rutinario de Hcto/ $\mathrm{Hb}$ no cambió el plan anestésico o detectó nuevas patologías ${ }^{16}$. Pensamos que la solicitud de $\mathrm{Hcto} / \mathrm{Hb}$ debe realizarse cuando exista historia sugerente o síntomas de anemia crónica ${ }^{7,8,17}$, patología de base que pudiera producir sangrado y/o anemia crónicos, y riesgo quirúrgico importante de hemorragia mayor intra o postoperatoria, en los cuales es vital tener un $\mathrm{Hcto} / \mathrm{Hb}_{\mathrm{basal}}{ }^{9}$.

\section{Pruebas de coagulación/Recuento de plaquetas}

El objetivo de solicitar estos exámenes es encontrar algún trastorno de coagulación que pudiera aumentar el riesgo de sangrado perioperatorio. Sin embargo, en la población quirúrgica general la prevalencia de algún trastorno de la coagulación hereditario es muy baja, siendo la coagulopatía no adquirida más común la enfermedad de Von Willebrand, la cual generalmente es asintomática, y con pruebas de coagulación (tiempo de protrombina, tiempo de tromboplastina activada) y recuento de plaquetas normales ${ }^{18}$. No existe evidencia suficiente que demuestre que el uso de pruebas de coagulación y/o recuento de plaquetas de rutina vaya a cambiar la morbimortalidad y manejo perioperatorio en pacientes asintomáticos ${ }^{8}$. En relación a la búsqueda activa por parte del cirujano de alteraciones de la coagulación que pudiesen afectar la cirugía, lo más importante a considerar es la historia clínica del paciente, el cual si no presenta clínica de alteraciones de la hemostasia (equimosis y/o sangrados mucocutáneos espontáneos, petequias sin causa aparente, epistaxis a repetición, gingivorragia frente a cepillado dental, hipermenorrea, etc.) no se le debiese solicitar pruebas de coagulación y/o recuento de plaquetas rutinariamente.

Tabla 1. Cirugía y riesgo de infarto o muerte de origen cardiovascular $^{14}$

Cirugía y riesgo de infarto o muerte de origen cardiovascular

Alto (Riesgo $>5 \%$ )

- Cirugía mayor de urgencia, especialmente en ancianos

- Cirugía aórtica u otra vascular mayor

- Cirugía vascular periférica

- Cirugía con cambios de volumen y/o sangre importante

Intermedio (Riesgo 1-5\%)

- Endarterectomía carotídea

- Cirugía de cabeza y cuello

- Cirugía intraperitoneal o intratorácica

- Cirugía ortopédica

- Cirugía prostática

Bajo (Riesgo < 1\%)

- Procedimientos endoscópicos

- Cirugía superficial

- Cirugía de cataratas

- Cirugía mamaria

Extraído de Manual de Anestesiología UC, 2006.

Tabla 2. Revised Cardiac Risk Index ${ }^{15}$

Revised Cardiac Risk Index
Cirugía de alto riesgo
Historia de cardiopatía isquémica (no revascularizada)
Historia de insuficiencia cardíaca
Historia de accidente cerebrovascular/Accidente isqué-
mico transitorio
Uso de insulina preoperatoria
Creatininemia $>2 \mathrm{mg}^{*} \mathrm{dl}^{-1}$

Extraído de Lee et al. Circulation 1999;100(10):1043-9. 
Recomendamos solicitar estos exámenes sólo si existe historia sugerente de alguna alteración en la hemostasia como las descritas anteriormente y si el paciente se encuentra en tratamiento anticoagulante o presenta alguna patología médica de base que pudiera alterar la hemostasia intra o postoperatoria, como una hemofilia ${ }^{8,17}$.

\section{Glucosa sanguínea}

Es conocido que una glicemia elevada, especialmente aquella $>180 \mathrm{mg}^{*} \mathrm{dl}^{-1}$, aumenta la morbimortalidad en el período perioperatorio, como también prolonga la estadía hospitalaria ${ }^{19}$, existiendo un significativo aumento en el riesgo de infección de herida operatoria ${ }^{20}$ y de falla cardíaca en el postoperatorio inmediato ${ }^{16}$. La incidencia de diabetes mellitus tipo 2 no diagnosticada en pacientes que se someterán a cirugía electiva es muy baja, siendo tan sólo un $0,5 \%{ }^{21}$. Por lo tanto, la recomendación actual es solicitar glicemia en ayunas preoperatoria en pacientes mayores de 75 años ${ }^{1}$, pacientes con historia sugerente de diabetes mellitus no diagnosticada (polifagia, polidipsia y baja de peso $)^{17} \mathrm{y} / \mathrm{o}$ usuarios de medicamentos que alteran la glucosa sanguínea, como los glucocorticoides 9 . En caso de pacientes diabéticos ya diagnosticados, la literatura médica recomienda solicitar hemoglobina glicosilada plasmática (HbA1c) por sobre una glicemia en ayunas aislada, ya que permite evaluar mejor el control glicémico de los últimos meses versus una muestra aislada de glucosa plasmática, que refleja el control glicémico sólo de las últimas horas preoperatorias ${ }^{9}$. En estos pacientes diabéticos conocidos, recomendamos solicitar HbA1c preoperatoria complementada por un hemoglucotest previo al ingreso a pabellón, repetido de forma seriada las primeras $12 \mathrm{~h}$ postoperatorias contadas desde el inicio de la cirugía.

\section{Función renal}

Johansson y $\operatorname{cols}^{8}$, realizaron un meta-análisis de 23 estudios relativos al tema, encontrando correlación positiva, estadísticamente significativa, entre alteraciones de la función renal preoperatorias y aumento de la morbimortalidad perioperatoria en 11 de ellos. Sin embargo, los 11 estudios mencionados incluían pacientes con enfermedades renales preexistentes lo que quita significancia a los hallazgos encontrados. De Sousa y cols ${ }^{13}$, estudiaron la función renal, con exámenes preoperatorios de rutina (nitrógeno ureico y creatinina plasmática) en pacientes sanos sometidos a cirugía electiva de bajo riesgo, no obteniendo ningún resultado anormal, por lo que no hubo ningún cambio de conducta quirúr- gica ni anestésica en el período perioperatorio. Una guía recomendó el uso de rutinario de creatinina plasmática en pacientes mayores de 40 años sometidos a cirugía electiva ${ }^{7}$. A raíz de estudios posteriores, la tendencia actual es no guiarse sólo por la edad del paciente para solicitar estudio preoperatorio de función renal, si no basarse también en la historia clínica y examen físico del paciente ${ }^{8,9}$. Actualmente, se debería solicitar nitrógeno ureico y creatininemia a todo paciente mayor de 65 años, el que presente alguna enfermedad sistémica que afecte la función renal o presente síntomas o signos clínicos sugerentes de enfermedad renal, como edema periférico, oliguria/poliuria, hematuria, etc ${ }^{1}$.

\section{Test embarazo}

La eficacia de un test de embarazo en orina está calculada en $99,8 \%$, con un valor predictivo positivo de aproximadamente $50 \%{ }^{8}$. El test no busca anormalidades, sino un diagnóstico que podría cambiar la conducta quirúrgica y anestésica en una paciente cursando un embarazo inicial. Muchas veces la historia clínica y el examen físico son inespecíficos para determinar un embarazo temprano, siendo necesario solicitar un test de embarazo a toda mujer en edad fértil y con actividad sexual reciente que será sometida a un procedimiento quirúrgico ${ }^{11}$. Está demostrado que mujeres jóvenes que dicen no estar embarazadas y que son sometidas a una intervención quirúrgica tienen un test de embarazo positivo en un $0,3 \%-2,2 \%{ }^{11}$. Es decir, aproximadamente 1 a 2 de cada 100 pacientes quirúrgicas en edad fértil y con actividad sexual reciente pueden estar embarazadas al momento de la intervención, por lo que creemos es recomendable solicitar este examen preoperatoriamente en esta población específica.

\section{Conclusiones}

El pilar fundamental de la evaluación preoperatoria de un paciente quirúrgico es la evaluación clínica a través de la anamnesis actual y remota, y el examen físico general y segmentario, los cuales deben ser realizados de forma completa y adecuada. La mayoría de las veces, la evaluación clínica es suficiente para establecer el riesgo perioperatorio y determinar los factores del paciente susceptibles de ser modificados durante el período preoperatorio, sin necesidad de recurrir a exámenes de apoyo complementarios ni interconsultas a especialistas. Sólo algunos hallazgos encontrados en la evaluación clínica preoperatoria pueden determinar la necesidad de solicitar exámenes de laboratorio, funcionales o de imágenes complementarios. 
Tabla 3. Exámenes sugeridos para pacientes sanos y asintomáticos

\begin{tabular}{|ccc|}
\hline Edad (años) & \multicolumn{2}{c|}{ Exámenes indicados } \\
& Hombres & Mujeres \\
$<40$ & Nada & Test embarazo \\
$40-49$ & EKG & Test embarazo \\
\hline $50-64$ & EKG & EKG \\
\hline $65-74$ & Hcto, EKG, BUN/Creat. \\
\hline$\geq 75$ & Hcto, EKG, Creat, Glicemia, RxTx o Ecocardiograma \\
\hline
\end{tabular}

Recomendación local Red de Salud UC, basada en Miller RD (ed) Anesthesia, vol 1, 6th ed. New York, Churchill Livingstone, 2005, p927-997.

Tabla 4. Recomendación de exámenes preoperatorios ${ }^{14}$

\begin{tabular}{|c|c|c|c|c|c|c|c|c|c|c|c|c|c|}
\hline Condición preoperatoria & \multicolumn{2}{|c|}{ Hb } & WBC & $\begin{array}{c}\text { TP/ } \\
\text { TTPK }\end{array}$ & $\begin{array}{c}\text { PLT, } \\
\text { TS }\end{array}$ & ELP & $\begin{array}{l}\text { Creat/ } \\
\text { BUN }\end{array}$ & Glicemia & $\begin{array}{c}\text { SGOT/ } \\
\text { FA }\end{array}$ & $\begin{array}{l}\mathbf{R x} \\
\mathbf{T x}\end{array}$ & ECG & $\begin{array}{c}\text { Test de } \\
\text { embarazo }\end{array}$ & $\mathbf{T} / \mathbf{S}$ \\
\hline \multicolumn{14}{|l|}{ Procedimiento quirúrgico: } \\
\hline - Con pérdida de sangre & $X$ & $\mathrm{X}$ & & & & & & & & & & & $X$ \\
\hline \multicolumn{14}{|l|}{ - Sin pérdida de sangre } \\
\hline Neonatos & $X$ & $\mathrm{X}$ & & & & & & & & & & & \\
\hline$<40$ años & & $\mathrm{X}$ & & & & & & & & & & & \\
\hline 40-49 años & & $\mathrm{X}$ & & & & & & & & & $\mathrm{H}$ & & \\
\hline 50-64 años & & $\mathrm{X}$ & & & & & & & & & $\mathrm{X}$ & & \\
\hline 65-74 años & $X$ & $\mathrm{X}$ & & & & & $\mathrm{X}$ & $\mathrm{X}$ & & $+/-$ & $\mathrm{X}$ & & \\
\hline$>75$ años & $\mathrm{X}$ & $\mathrm{X}$ & & & & & $\mathrm{X}$ & $\mathrm{X}$ & & $+/-$ & $\mathrm{X}$ & & \\
\hline Enfermedad cardiovascular & & & & & & & $\mathrm{X}$ & & & $\mathrm{X}$ & $\mathrm{X}$ & & \\
\hline Enfermedad pulmonar & & & & & & & & & & $\mathrm{X}$ & $X$ & & \\
\hline Malignización & $\mathrm{X}$ & $\mathrm{X}$ & $*$ & $*$ & & & & & & $\mathrm{X}$ & & & \\
\hline Radioterapia & & & $\mathrm{X}$ & & & & & & & $\mathrm{X}$ & $\mathrm{X}$ & & \\
\hline Enfermedad hepática & & & & $\mathrm{X}$ & & & & & $\mathrm{X}$ & & & & \\
\hline Exposición a hepatitis & & & & & & & & & $\mathrm{X}$ & & & & \\
\hline Enfermedad renal & $\mathrm{X}$ & $\mathrm{X}$ & & & & $\mathrm{X}$ & $\mathrm{X}$ & & & & & & \\
\hline Alteración homeostasis & & & & $X$ & $X$ & & & & & & & & \\
\hline Diabetes & & & & & & $\mathrm{X}$ & $\mathrm{X}$ & $\mathrm{X}$ & & & $\mathrm{X}$ & & \\
\hline Fumador $>20$ paq/año & $\mathrm{X}$ & $\mathrm{X}$ & & & & & & & & $\mathrm{X}$ & & & \\
\hline Embarazo posible & & & & & & & & & & & & $\mathrm{X}$ & \\
\hline Uso de diuréticos & & & & & & $X$ & $\mathrm{X}$ & & & & & & \\
\hline Uso de digoxina & & & & & & $\mathrm{X}$ & $\mathrm{X}$ & & & & $\mathrm{X}$ & & \\
\hline Uso de esteroides & & & & & & $X$ & & $X$ & & & & & \\
\hline Uso de anticoagulantes & $\mathrm{X}$ & $\mathrm{X}$ & & $\mathrm{X}$ & & & & & & & & & \\
\hline Enfermedad de SNC & & & $\mathrm{X}$ & & & & $X$ & $\mathrm{X}$ & $\mathrm{X}$ & & $X$ & & \\
\hline
\end{tabular}

Recomendación local Red de Salud UC, basada en Concha y De la Cuadra (ed) Manual de Anestesiología, Primera edición, Santiago: Ediciones Universidad Católica de Chile; 2006, p209-228. Nota: Se debe usar el juicio clínico en aquellas enfermedades que no aparecen en esta lista. Símbolos: X: necesario; H: hombres; M: mujeres; +/-: quizás necesario; *: necesario en leucemias. Abreviaciones: Hb: hemoglobina; WBC: recuento de blancos; TP: tiempo de sangría; TTPK: tiempo parcial de tromboplastina; PLT: recuento de plaquetas; TS: tiempo de sangría; ELP: electrolitos plasmáticos ( $\mathrm{Na}, \mathrm{K}, \mathrm{Cl})$; $\mathrm{Crea} / \mathrm{BUN}$ : Creatinina y nitrógeno ureico; SGOT/FA: transaminasa glutámico-oxalacética y fosfatasas alcalinas; ECG: electrocardiograma; T/S: Tipificación sanguínea y screening anticuerpos inesperados; SNC: sistema nervioso central. 
En concordancia con el ASA Task Force, concluimos que los exámenes preoperatorios no deben ser solicitados de forma rutinaria y menos indiscriminadamente. Los test preoperatorios debieran ser solicitados en base a la condición clínica actual del paciente y al tipo de cirugía a la que se someterá, realizando una solicitud racional, selectiva y costoefectiva de aquellos exámenes que efectivamente nos permitan mejorar los resultados del período periquirúrgico, disminuyendo la mortalidad y morbilidad perioperatorias. Los exámenes preoperatorios sólo son un complemento en la evaluación preoperatoria, por lo que no deben ser utilizados como tamizaje por el cirujano general o subespecialista.

La literatura médica sugiere realizar algunos exámenes preoperatorios de laboratorio y/o de imágenes "básicos" o "de rutina", según las características clínicas (sintomatología y/o signología presentes) y patologías crónicas del paciente. Así, un paciente sin patología crónica de base (sano) y carente de síntomas y signos (asintomático) va a necesitar de ciertos exámenes prequirúrgicos dependiendo de su sexo y edad, como lo muestra la Tabla 3.

Por otro lado, pacientes con patologías crónicas conocidas en tratamiento y control van a necesitar de ciertos exámenes de laboratorio y/o imágenes, dependiendo de la o las enfermedad (es) crónica (s) que padezca (n). Así, un paciente con una insuficiencia renal crónica debería ser estudiado según las repercusiones en los órganos blancos que presente (aparato cardiovascular, sistema neurológico, sistema nefrológico, etc.), solicitando preoperatoriamente función renal (BUN/Creatininemia), electrolitos plasmáticos (kalemia especialmente), electrocardiograma de reposo y hematocrito (eventualmente hemograma/VHS), según resume la Tabla 4.

\section{Referencias}

1. Roizen M. Preoperative evaluation. In: Miller RD (ed) Anesthesia, vol 1, 6th ed. New York, Churchill Livingstone, 2005, p927-97.

2. Xuan Thanh N, Rashiq S, Jonsson E. Routine preoperative electrocardiogram and chest $\mathrm{x}$-ray prior to elective surgery in Alberta, Canada. J Can Anesth. 2010;57:12733.

3. Olsen D, Kane R, Proctor P. A controlled trial of multiphasic screening. N Engl J Med. 1976;294:925.

4. Durbridge T, Edwards F, Edwards R, Atkinson M. Evaluation of benefits of screening tests done immediately on admission to hospital. Clin Chem. 1976;22:968-71.

5. Narr B, Hansen T, Warner M. Preoperative laboratory screening in healthy Mayo patients: Cost-effective elimination of tests and unchanged outcomes. Mayo Clin Proc. 1991;66:155-9.

6. Munro J, Booth A, Nicholl J. Routine preoperative testing: a systematic review of the evidence. Health Technol Assess 1997;1:1-62.

7. Institute for Clinical Systems Improvement. Health care guideline: preoperative evaluation. 10th ed. Bloomington, Minn.: Institute for Clinical Systems Improvement; 2012.

8. Johansson T, Fritsch G, Flamm M, Hansbauer B, Bachofner N, Mann E, et al. Effectiveness of non-cardiac preoperative testing in non-cardiac elective surgery: a systematic review. Br J Anaesth. 2013;110:926-39.

9. Feely M, Collins C, Daniels P, Kebede E, Jatoi A, Mauck K. Preoperative Testing Before Noncardiac Surgery: Guidelines and Recommendations. Am Family Physician 2013;87:414-25.

10. American Society of Anesthesiologists Task Force on Preanesthesia Evaluation. Practice advisory for preanesthesia evaluation. Anesthesiology 2002;96:485-96.

11. Hepner D. The role of testing in the preoperative evaluation. Cleveland Clinic J Med. 2009;76:22-7.

12. Dripps RD. New classification of physical status. American Society of Anesthesiologists. Anesthesiology 1963;24:111.

13. De Sousa D, Marques R, Nogueira M, Fernandes V, Vieira A, Santana E. Relevancia de los Exámenes de Rutina en Pacientes de Bajo Riesgo Sometidos a Cirugías Menores. Rev Bras Anestesiol. 2013;63:197-201.

14. Dagnino J, Concha M: Evaluación preoperatoria. En: Concha M, De la Cuadra JC (ed): Manual de Anestesiología, Primera edición, Santiago: Ediciones Universidad Católica de Chile; 2006, p209-28.

15. Lee T, Marcantonio E, Mangione C, Thomas E, Polanczyk C, Cook E, et al. Derivation and prospective validation of a simple index for prediction of cardiac risk of major noncardiac surgery. Circulation 1999;100:1043-9.

16. Gnocchi C, Risso J, Khoury M, Torn A, Noel M, Baredes $\mathrm{N}$, et al. Application of a preoperative evaluation model in patients undergoing elective abdominal surgery. Medicine 2000;60:125-34.

17. De Hert S, Imberger G, Carlisle J, Diemunsch P, Fritsch G, Moppett I. et al. Task Force on Preoperative Evaluation of the Adult Noncardiac Surgery Patient of the European Society of Anaesthesiology. Preoperative evaluation of the adult patient undergoing non-cardiac surgery: guidelines from the European Society of Anaesthesiology. Eur J Anaesth. 2011;28:684-722.

18. Dempfle CE. Perioperative coagulation diagnostics. Anaesthesist J. 2005;54:167-78.

19. Clement S, Braithwaite S, Magee M, Ahmann A, Smith E, Schafer R, et al. Management of diabetes and hyperglycaemia in hospitals. Diabetes Care 2004;27:553-91.

20. Ata A, Lee J, Bestle SL, Desemone J, Stain SC. Postoperative hyperglycemia and surgical site infection in general surgery patients. Arch Surg. 2010;145:858-64.

21. Smetana G, Macpherson D. The case against routine preoperative laboratory testing. Med Clin North Am. 2003;87:7-40. 